Proc. Indian Acad. Sci. (Chem. Sci.), Vol. 106, No. 5, October 19,4, pp. 1177-1189.

(C) Printed in India.

\title{
Binding of active site directed ligands to phospholipase A2: Implications on the molecular constraints and catalytic mechanism
}

\author{
K SESHADRI $^{1}$, SARASWATHI VISHVESHWARA ${ }^{*, 1}$ and \\ MAHENDRA K JAIN ${ }^{1,2}$ \\ ${ }^{1}$ Molecular Biophysics Unit, Indian Institute of Science, Bangalore 560012, India \\ ${ }^{2}$ Department of Chemistry and Biochemistry, University of Delaware, Newark, DE 19716, \\ USA
}

\begin{abstract}
Molecular constraints for the localization of active site directed ligands (competitive inhibitors and substrates) in the active site of phospholipase A2 (PLA2) are characterized. Structure activity relationships with known inhibitors suggest that the head group interactions dominate the selectivity as well as a substantial part of the affinity. The $a b$ initio fitting of the amide ligands in the active site was carried out to characterize the head group interactions. Based on a systematic coordinate space search, formamide is docked with known experimental constraints such as coordination of the carbonyl group to $\mathrm{Ca}^{2+}$ and hydrogen bond between amide nitrogen and ND1 of His48. An optimal position for a bound water molecule is identified and its significance for the catalytic mechanism is postulated. Unlike the traditional "pseudo-triad" mechanism, the "Ca-coordinatedoxyanion" mechanism proposed here invokes activation of the catalytic water to form the oxyanion in the coordination sphere of calcium. As it attacks the carbonyl carbon of the ester, a near-tetrahedral intermediate is formed. As the second proton of the catalytic water is abstracted by the ester oxygen, its reorientation and simultaneous cleavage form hydrogen bond with ND1 of His48. In this mechanism of esterolysis, a catalytic role for the water co-ordinated to $\mathrm{Ca}^{2+}$ is recognised.
\end{abstract}

Keywords. PLA2; inhibitors; catalytic mechanism; molecular modelling; transition state.

\section{Introduction}

Interfacial catalysis is an obligatory mode of action for the enzymes whose natural substrates are present at the organized interfaces. For example, most, if not all, enzymes of lipid metabolism must access the substrate during the catalytic turnover from a bilayer of micellar interface. The concentration of the solitary substrate monomers in the aqueous phase (defined as critical micelle concentration or $\mathrm{cmc}$ ) is less than $100 \mathrm{pM}$; and at higher concentrations excess amphiphiles form organized interfaces (Jain 1988).

The current understanding of interfacial catalysis is based predominantly on studies with secreted phospholipase A2 (PLA2) present in venoms, pancreatic juices and inflammatory exudates. As shown in the kinetic scheme in figure 1, as a prelude to

\footnotetext{
*For correspondence
}

Abbreviations. cmc, critical micelle concentration; PLA2, $14 \mathrm{kD}$ secreted phospholipase A2; ND1 of His48, nitrogen at the delta position of the imidazole ring of histidine-48; NE2, nitrogen at the epsilon position of histidine-48. 


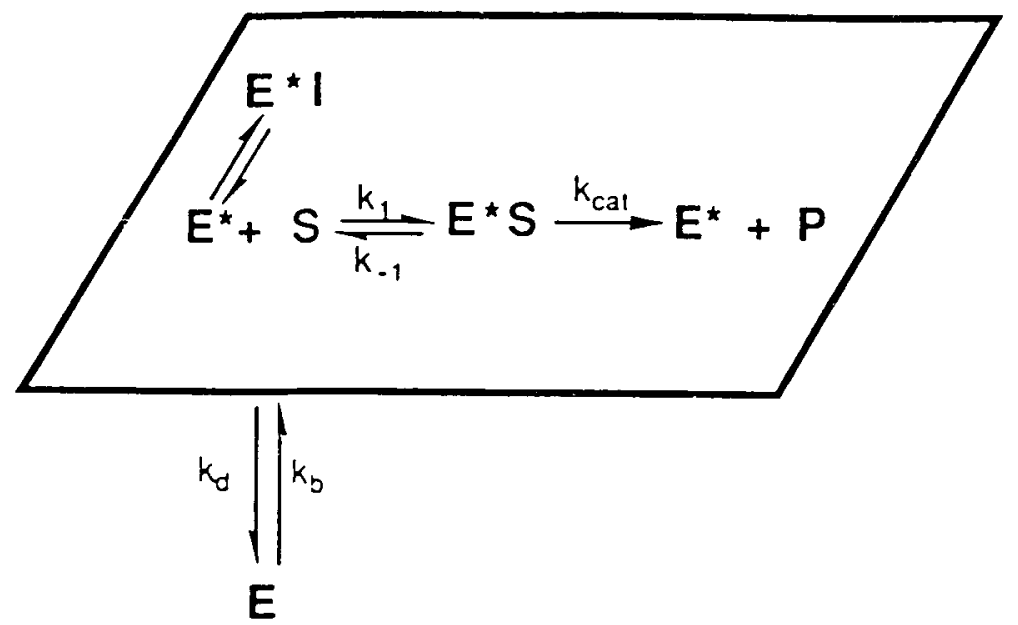

Figure 1. A scheme to accommodate key features of interfacial catalysis by PLA2. The species ( $E^{*}$, enzyme; $I$, inhibitor; $S$, substrate; $P$, products) shown in the box plane are in or bound to the bilayer. The enzyme in the aqueous phase is shown as $E$. During the steady state, $E$ is in equilibrium with the enzyme in the interface $\left(E^{*}\right)$, where according to the Michaelis-Menten formalism, $E^{*}$ brings about catalytic turnover by the steps shown in the box. Factors that regulate catalysis would modulate the steps in the box, while a shift in the $E$ to $E^{*}$ equilibrium would modulate the apparent rate of catalytic turnover by increasing or decreasing the fraction of the total enzyme in the catalytically active $E^{*}$ form.

catalytic turnover the water-soluble PLA2 must bind to the interface where they hydrolyze the $s n$-2-ester linkage of the naturally occuring $s n$-3-glycero-phospholipids. Due to their low cmc, not only the substrate but also the products of hydrolysis remain at the interface. Also the bilayer organization of phospholipids is retained even after virtually all the phospholipids at the interface have been hydrolyzed. A critical review of the relevant background literature (Jain and Berg 1989) and of the evidence leading to a quantitative elucidation of the steps shown in the kinetic scheme (Berg et al 1991) is reviewed elsewhere (Jain et al 1994). This scheme is an adaptation of the Michaelis-Menten formalism to accomodate key features of lipolysis at interfaces. The minimal kinetic scheme shown in figure 1 is justified because the chemical step is not reversible and that this step is rate-limiting (i.e. slower than all the subsequent steps including the product release).

\subsection{Consequences of the $E$ to $E^{*}$ step in the kinetic scheme}

A key feature of the kinetic scheme shown in figure 1 is that the substrate concentration has two separate levels of meaning for the rate and equilibria that control the overall catalytic turnover. First, the fraction of the enzyme in the interface depends upon the bulk concentration of the substrate, expressed for convenience in the units of moles per litre. It is an approximate measure of the surface of the substrate available for the encounter of the free enzyme and thus it controls the $E$ to $E^{*}$ step. Only the $E^{*}$ form is responsible for the catalytic turnover. Secondly, the substrate concentration that the bound enzyme $\left(E^{*}\right)$ 'sees' at the interface is the number density of the substrate molecules expressed conveniently in units of mole fraction with a maximum possible 
value of 1 . Thus, the mole fraction of the substrate, or for that matter of any other ligand, controls the equilibrium for the formation of the Michaelis-Menten complex at the interface, i.e. the $E^{*}+S$ to $E^{*} S$ equilibrium deterinines the probability of occupancy of the catalytic site of the enzyme at the interface and therefore the effective rate of the product formation. Thus, the fraction of the total enzyme in the $E^{*}, E^{*} S$, $E^{*} I$ and $E^{*} P$ forms, ultimately determines the effective observed rate of hydrolysis.

\subsection{Kinetic basis for interfacial catalysis}

The kinetic scheme in figure 1 explicitly distinguishes binding of the enzyme to the interface from the steps of the catalytic turnover. Besides the fraction of the bound enzyme, factors that control the microscopic steady state are also critically important for the interpretation of the observed reaction progress. In order to meaningfully interpret even some of the simplest time courses of reaction progress by interfacial catalysis, it is necessary to take into consideration the variables that control the availability of the substrate to the enzyme at the interface where the catalytic turnover occurs. A major difficulty in describing the time course of the reaction progress comes from the fact that the residence time of the enzyme at the interface cannot be readily ascertained, i.e. how much time an enzyme molecule spends in the aqueous phase and how much at the interface where catalysis occurs. To appreciate the nature, magnitude and extent of the underlying complexity consider an ensemble of phospholipid vesicles, each containing say about 10,000 phospholipid molecules in the, outer monolayer. After the addition of PLA2, the reaction begins as the enzyme is equilibrated between the aqueous phase and the interface. The kinetic description of the reaction progress would be rather straightforward if the enzyme binds to and dissociates from the interface during each catalytic turnover cycle, i.e. if the rate constants $k_{b}$ and $k_{d}$ are a part of the catalytic turnover cycle by the steps shown in the box in figure 1 . Unfortunately, the rate constants for the interfacial binding and desorption steps are quite small compared to the time for a single catalytic turnover (Jain et al 1988). On the other hand, at a zwitterionic interface the rate of desorption is rapid compared to the time course of the overall reaction progress for the hydrolysis of a vesicle. Moreover, the values of $k_{b}$ and $k_{d}$ depend upon the composition of the interface including the mole fraction of the products of hydrolysis. Thus, depending upon the residence time of the enzyme on an interface, the mole fraction of the substrate it "sees" at any given instance of time could be significantly different from the bulk-averaged mole fraction in the reaction mixture.

The problem of kinetic interpretation becomes even more complex if one considers the realistic possibility that the enzyme desorbed after a few catalytic turnovers encounters a vesicle that has previously encountered an enzyme molecule. It is also possible that the desorbed enzyme can encounter a vesicle that has not 'seen' the enzyme before. The net result of such 'scrambling' would be that at any given time point during progress of the reaction different enzyme molecules will be present in different microenvironments. Such a departure from a microscopic steady-state precludes the possibility of "ensemble averaging" the behaviour of all the enzyme molecules in the reaction mixture at each time point during the reaction progress. In short, if the enzyme is able to hop from one interface to another during the progress of the reaction (figure 2), violation of the microscopic steady-state condition by the factors outlined above would make it impossible to interpret the reaction progress 

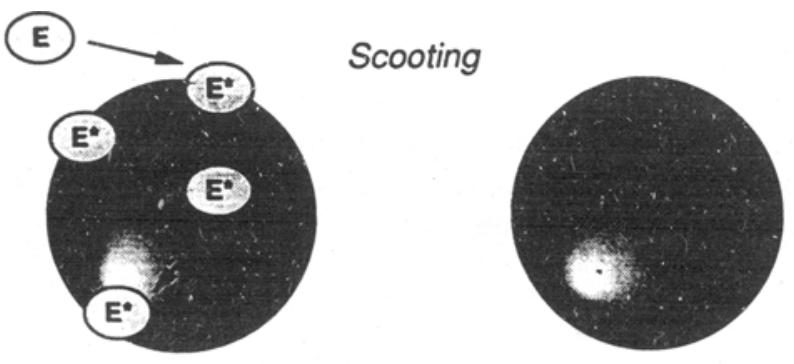

(a)

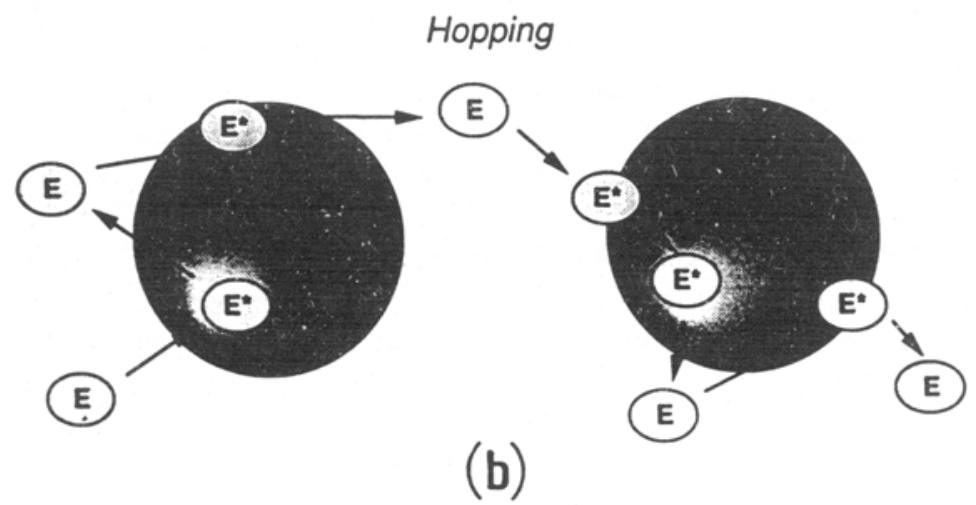

Figure 2. Schematic illustration of some key features of interfacial catalysis on vesicles in the scooting and the hopping modes. (a) In the scooting mode when the vesicle to enzyme ratio is $>5$, there is at most one enzyme per enzyme-containing vesicle. Due to the high affinity $\left(K_{D}<0.1 \mathrm{pM}\right)$ of PLA2 for DMPM vesicles, the bound enzyme $\left(E^{*}\right)$ does-not leave the vesicle even when all of the substrate in the outer monolayer of the target vesicles is hydrolyzed. Therefore, excess vesicles are not hydrolyzed by the enzyme added initially unless the vesicles are allowed to fuse, the bound enzyme is forced to undergo intervesicle exchange, or the excess vesicles are hydrolyzed by adding excess enzyme so that there is at least one enzyme per vesicle. (b) On the other hand, during catalysis in the hopping mode, the enzyme desorbs from the vesicle surface, and thus all vesicles are ultimately hydrolyzed even if the vesicle to enzyme ratio is $\gg 1$.

only in terms of the primary rate constants shown in the box in figure 1. Such limitations also apply for the hydrolysis of mixed micelles where the substrate on the enzyme-containing micelle is rapidly depleted and therefore the observed rate is limited by the rate of replenishment (Jain et al 1993a).

\subsection{Interfacial catalysis in the scooting mode}

The difficulties created by the exchange of the enzyme with excess vesicles are circumvented by monitoring interfacial catalysis in the scooting mode where the bound enzyme does not leave the interface. Under suitable conditions the enzyme does not leave the interface not only during the catalytic turnover cycle but also during the course of the observed reaction progress (which may be of the order of several minutes). Results of the catalytic turnover in the scooting mode can be 
rigorously interpreted because the following conditions are satisfied. First, in a reaction mixture containing an excess (in accordance with the Poisson distribution) of vesicles it is possible to assure that each enzyme containing vesicle contains at most one enzyme per vesicle. Second, by using vesicles of narrow monomodal distribution of sizes it is possible to assure that all enzyme molecules experience identical environment during the whole time course of the reaction progress. Third, by using large vesicles it is possible to measure the 'initial rate' from the reaction progress where the microscopic steady state concentration of the substrate seen by each enzyme molecule remains not only the same but also constant at mole fraction $=1$. This last condition is also fulfilled, if by some means (fusion, exchange or mediated transfer) lipid molecules are rapidly transferred from excess vesicles to the enzyme containing vesicles.

In fact, by judicious choice of the experimental conditions we have been able to obtain and interpret not only the initial rate but also the whole reaction progress curve in terms of the primary rate and equilibrium constants. As reviewed elsewhere (Jain et al 1994), with additional independent measurements it is possible to obtain virtually all the primary rate and equilibrium parameters that describe the interfacial kinetic behaviour of secreted PLA2 from several different sources and their sitedirected mutants. This approach has also provided protocols to establish substrate specificity, the role of calcium as a cofactor, the basis for interfacial activation, and the kinetic effect of inhibitors on the reaction progress. It may be emphasized that such fundamental information about interfacial catalysis has not been obtained by other assay protocols used for PLA2 or any other interfacial enzyme.

\subsection{Inhibitors of interfacial catalysis}

The primary focus of this article is to understand the molecular constraints for the interaction of active site-directed ligands of PLA2. Scooting mode kinetics provides an ideal basis for monitoring inhibition of interfacial catalysis by PLA2 (Jain et al $1986,1989,1991,1992,1993 b$ ). This has not been possible with any of the other assay methods used in the literature because in such assays any agent that perturbs the $E$ to $E^{*}$ equilibrium would also decrease the observed rate of hydrolysis. Such "falsepositive" inhibitors include mepacrine, aristolochic acid, indomethacin, local anesthetics, and a whole range of amphiphilic peptides and proteins. In fact, the problem of characterization of inhibitors of interfacial catalysis has been so acute that virtually all the inhibitors of PLA2 reported before 1986 are not specific competitive inhibitors. Such kinetic artefacts are known for other interfacial enzymes, which have hampered progress towards isolation and characterization of naturally occurring inhibitors of virtually all interfacial enzymes.

The scooting mode protocol for the assay of PLA2 inhibitors does not give a false-positive. As summarized in figure 3, by this procedure several specific competitive inhibitors of PLA2 have been identified. The active site directed nature of these inhibitors has been characterized by additional criteria: (a) Only one of the enantiomers is inhibitory; (b) They bind to the catalytic site as demonstrated by the fact that the rate of alkylation of the catalytic residue His 48 decreases well over 50 -fold in the presence of the inhibitors; (c) calcium is an essential cofactor for PLA2 as demonstrated by the fact that the cation is required for the binding of the inhibitor to the active site of the enzyme; (d) the affinity of the $E^{*}$ form of the enzyme for the 
<smiles>CC(=O)N[C@@H](CO)COP(=O)([O-])OCC[N+](C)(C)C</smiles>

(a)<smiles>[R]C[C@H](COCC(F)(F)F)OP(=O)([O-])OC</smiles>

(c)<smiles>[NH3+]COP(=O)([O-])OC[C@H](CO)OP(=O)([O-])[O-]</smiles>

(b)<smiles>[R]C(N)=O</smiles>

(d)<smiles>[R]C(=O)NC</smiles>

(e)

Figure 3. Structures of catalytic site-directed inhibitors of PLA2 ( $R$ represents fatty acyl or alkyl chain). (a) The $s n$-2-amide analog of $s n$-3-phospholipid; (b) the $s n-2$-phosphonate analog of sn-3-phospholipid; (c) the sn-2-phosphate and phosphonate derivatives of a triglyceride; (d) fatty acyl amide; and (e) its $\mathrm{N}$-methyl derivate.

active site directed ligands is higher than the affinity of the $E$ form; (e) the X-ray crystal structures of PLA2 with two transition state mimics of type A and B in figure 3 at the active site are consistent with the localization of these ligands at the active site; (f) structure activity correlation shows that the binding of the inhibitor to the $E^{*}$ form is dominated by the head group interactions, although effects related to the partitioning of the inhibitor into the interface depend on the chain length.

Of the various inhibitors of PLA2 shown in figure 3, primary amides of fatty acids are particularly intriguing in the sense that the inhibitory potency of these simple molecules is of the same order of magnitude as that of the "transition state mimics" of the substrate.

\section{Modelling of the ligand at the active site}

\subsection{Binding of formamide to PLA2}

In order to further understand the mode of interaction of fatty acyl amides to the active site of secreted PLA2, we undertook computer modelling studies. For this purpose, the X-ray crystal structure (Scott et al 1990; Thunnissen et al 1990) of the native PLA2 and its complex (with the type A or B inhibitors) were considered to set up the initial binding constraints. The following constraints were also imposed for detailed docking of formamide into the active site. (i) The distance ' $q$ ' (Ca...O) between the calcium and the carbonyl oxygen of the amide was fixed between $2.5 \AA$ to $2.6 \AA$. (ii) The distance ' $p$ ' (ND1-His48...N(amide)) was maintained at hydrogenbonding distance $(3.0 \AA)$ as shown in figure $4 \mathrm{a}$. The Eulerian angles $(\phi, \theta, \psi)$ used to describe a rigid body motion of the ligand in the active site (Goldstein 1980) are shown in figure $4 \mathrm{~b}$. The parameter ' $\alpha$ ' describes the orientation of the amide with respect to His 48 and ' $\omega$ ' can take values of $0^{\circ}$ and $180^{\circ}$ for a planar amide. Standard internal parameters (Ramachandran and Sasisekharan 1968) are chosen for amide. 

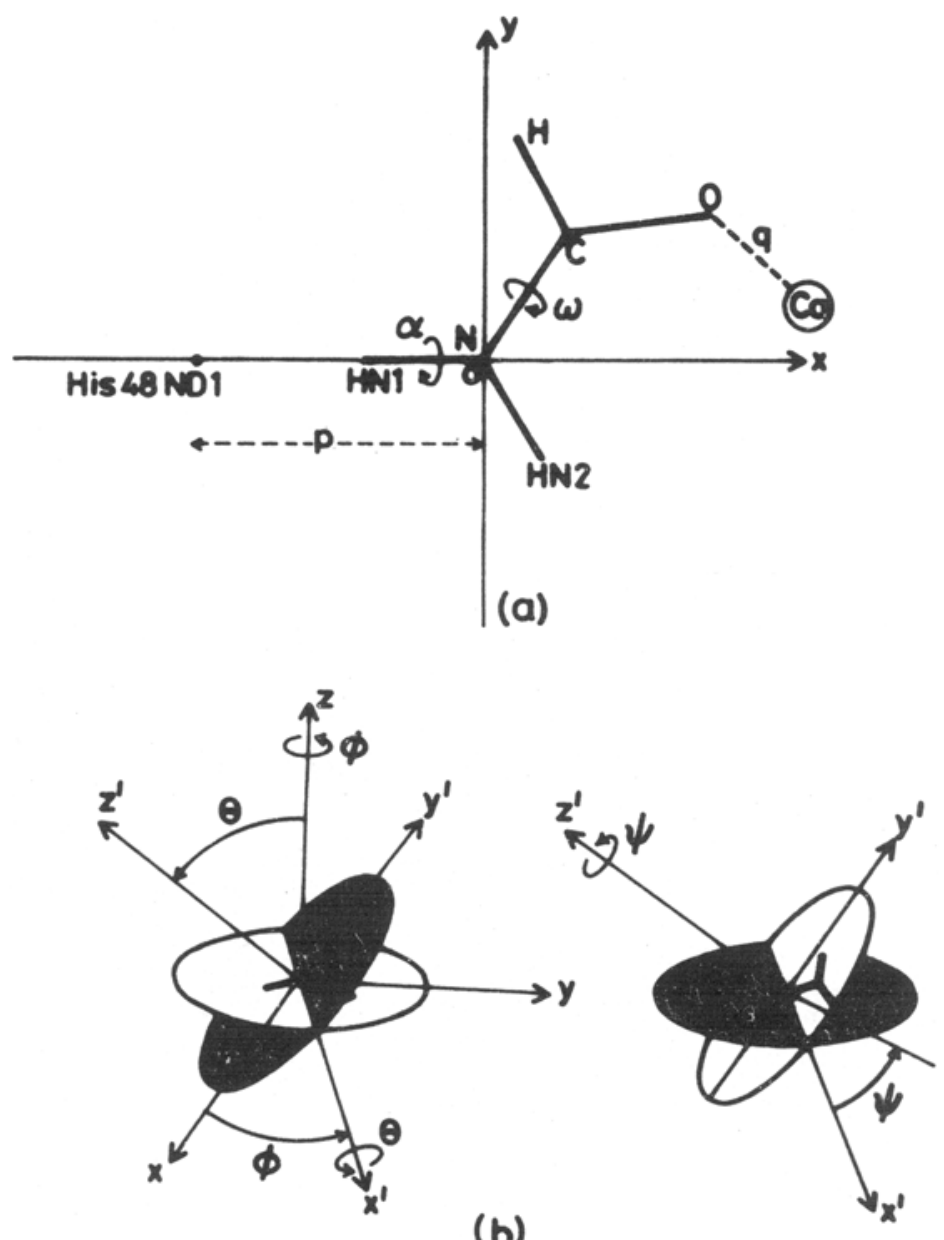

(b)

Figure 4. The geometric construction of the amide in the active site of PLA2. (a) Amide plane positioned between His48 ND1 and $\mathrm{Ca}^{2+}$. (b) Eulerian angles that enable the amide plane span the Cartesian space around the origin fixed at $\mathbf{N}$ (see text for description of the parameters).

The following procedure was used to explore all possible modes of amide binding in the active site of PLA2 and to narrow it down to a few allowed positions. (i) The conformational space of $(\phi, \theta, \psi)$ was systematically explored by varying in steps of $10^{\circ}$. The parameter ' $\alpha$ ' was varied in steps of $60^{\circ}$ and two values of ' $\omega$ ' $\left(0^{\circ}\right.$ and $\left.180^{\circ}\right)$ were explored. This gave $279936(36 \times 18 \times 36 \times 6 \times 2)$ possible orientations, (ii) All these orientations were tested for 'contact' between the amide atoms and the PLA2 atoms (Ramachandran and Sasisekharan 1968; Sekharadu 1985; Balaji 1990). The orientations of the amide devoid of any steric hindrance within the 'overlap limit' of $1.0 \AA$ were identified. This procedure gave less than 200 sterically allowed conformations. (iii) Three-dimensional $(\phi, \theta, \psi)$ contact map's were generated for all the six $\alpha$ values $\left(0^{\circ}, 60^{\circ}, 120^{\circ}, 180^{\circ}, 240^{\circ}\right.$ and $\left.300^{\circ}\right)$. An example of a contant map for $\alpha=240^{\circ}$ and $\omega=180^{\circ}$ is shown in figure 5 which indicates five contact-free regions. Twenty distinct contact-free regions were obtained from the contact maps of all six 


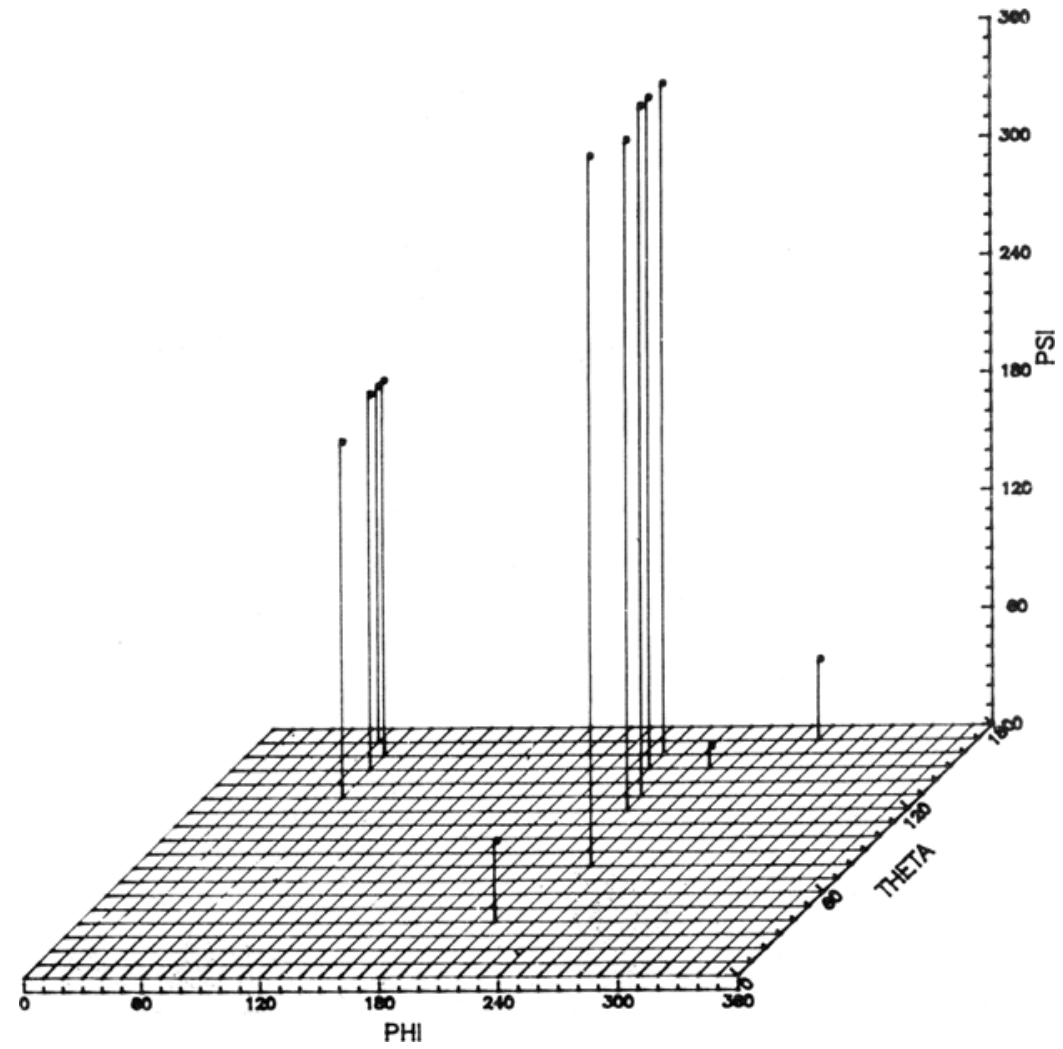

Figure 5. A three-dimensional contact $(\phi, \theta, \psi)$ map for $\alpha=240^{\circ}$ and $\omega=180^{\circ}$.

Table 1. Representative orientations (in degrees) selected from the contact maps $^{-1}$.

\begin{tabular}{rrrrcrrr}
\hline$\alpha$ & $\phi$ & $\theta$ & $\psi$ & $\alpha$ & $\phi$ & $\theta$ & $\psi$ \\
\hline 60 & 30 & 30 & 200 & 240 & 70 & 150 & 190 \\
60 & 50 & 140 & 170 & 240 & 210 & 40 & 40 \\
60 & 340 & 150 & 80 & 240 & 220 & 120 & 340 \\
120 & 40 & 60 & 210 & 240 & 280 & 170 & 40 \\
120 & 50 & 110 & 190 & 300 & 220 & 120 & 330 \\
120 & 50 & 160 & 180 & 300 & 230 & 20 & 360 \\
120 & 120 & 170 & 240 & 300 & 250 & 170 & 360 \\
120 & 280 & 130 & 40 & 360 & 50 & 50 & 190 \\
180 & 60 & 90 & 200 & 360 & 60 & 100 & 170 \\
180 & 240 & 100 & 360 & 360 & 240 & 90 & 350 \\
\hline 1
\end{tabular}

${ }^{1}$ The angular parameters $\alpha, \phi, \theta$ and $\psi$ are described in figure 4 .

$\alpha$ values. The parameters of these orientations are listed in table 1 and a stereoview of a few of these allowed amide orientations along with $\mathrm{Ca}^{2+}$ and His48 is given in figure 6.

In short, the $a b$ initio method of docking amide by rigid body rotation and ligand conformational search places the amide in 20 possible contact-free orientations which 

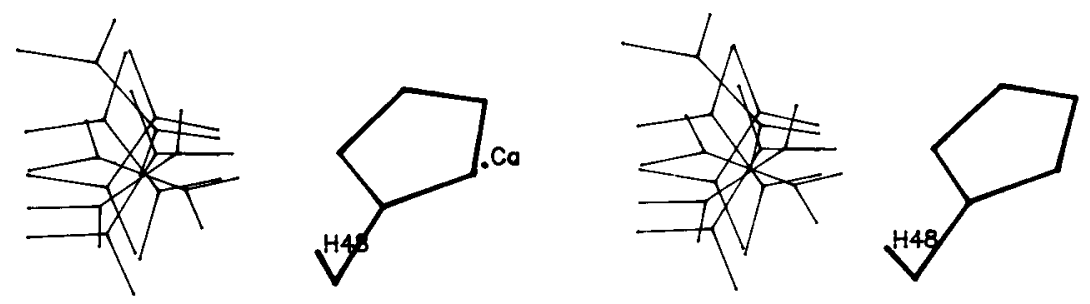

Figure 6. Stereoscopic view of some allowed orientations of amide that have simultaneous interaction with His48 ND1 and coordination with $\mathrm{Ca}^{2+}$.

coordinate with $\mathrm{Ca}^{2+}$ and are also at hydrogen-bonding distance from ND1 of His 48. It is interesting to note that the hydrogen bonding of amide $\mathrm{H} 1$ to His 48 was possible only with ND1; the amide hydrogen bonding to NE2 was not suitably placed for coordination with $\mathrm{Ca}^{2+}\left(\mathrm{NE} 2 . . \mathrm{Ca}^{2+}\right.$ distance $\left.=8.59 \AA\right)$. As described next, additional constraints for the localization of the displaced water molecule led to a single unique position for the amide.

\subsection{Water coordination}

In the native structure of PLA2, two water molecules are coordinated to the $\mathrm{Ca}^{2+}$ ion. One of them is in the pentacoordination plane formed by carbonyls of Gly 30 , Gly32 and OD1 and OD2 of Asp49. The apical positions are occupied by the second water molecule and the carbonyl of Tyr28. These water molecules were not included in the amide docking exercise. It is interesting to see that during the $a b$ initio docking the amide oxygen has occupied a position very close to the water position in the pentacoordination plane.

A position for the water was searched to see if the water of the native enzyme is totally expelled from the region or only displaced. A grid was constructed enclosing the $\mathrm{Ca}^{2+}$, amide and carbonyl oxygen of Tyr28. Grid points were generated with a step size of $0.2 \AA$ for all the 20 orientations given in table 1 . Oxygen of the water molecule was placed at every grid point and its distance from $\mathrm{Ca}^{2+}$, the amide oxygen, the amide $\mathrm{H} 2(\mathrm{~N})$, the carbonyl oxygen of Tyr28, and the carboxylate oxygens of Asp49 was monitored. The water positions which coordinated with calcium were analysed for hydrogen bonds with the active site groups and with the amide oxygen. Of the 20 allowed amide positions, only the 14th orientation (table 1) and a very few water positions satisfied the coordination ion and maximal hydrogen bonding. The stereoscopic representation and a schematic diagram indicating the evaluated distances for this orientation are given in figures $7 a$ and $b$, respectively. The oxygen of water is at $2.75 \AA$ and $1.93 \AA$ from $\mathrm{Ca}^{2+}$ and amide proton (H2) respectively. The protons of this water can be oriented to form hydrogen bonds with carbonyl oxygens of the amide inhibitor and Tyr28. Particularly striking is the fact that both the amide oxygen and the displaced water is within the coordinating distance of $2 \cdot 8 \AA$ from calcium, resulting in a total of eight groups liganded to the metal ion. The distances and the geometry given in figure $7 \mathrm{~b}$ are consistent with these features and are being further refined by energy minimization studies. The consequences of the presence of this water molecule as an additional ligand coordinated to calcium are elaborated in subsequent sections. 

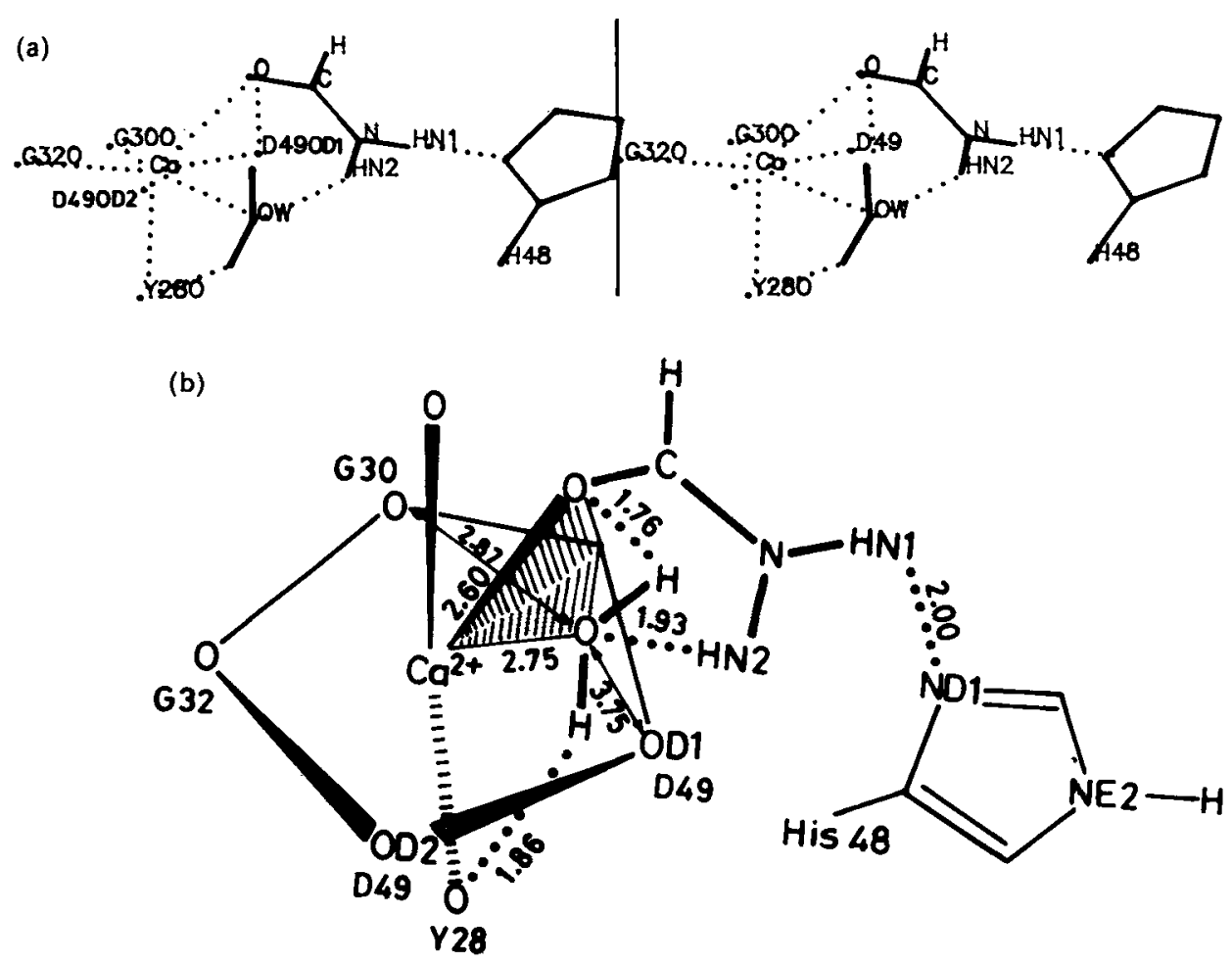

Figure 7. (a) Stereoscopic view of amide and water docked with maximal interactions in the active site of PLA2. (b) Schematic diagram showing the distances involved in (a).

\subsection{Binding of $N$-methylformamide}

The formamide hydrogen $\mathrm{H} 2$ was replaced by $-\mathrm{CH}_{3}$ group and the 20 allowed positions given in table 1 were again tested for contacts. The search revealed that methyl substitution did not have any effect on these allowed orientations. Hence, the experimentally observed affinity of formamide and its loss in $\mathrm{N}$-methyl formamide are not due to its steric effect on interaction with the enzyme. Although $\mathrm{N}$-methylation of amide had no effect in reducing contact-free region, the methyl group would not have allowed the above identified water (equatorial) molecule to take its position in the coordination sphere for $\mathrm{Ca}^{2+}$. Further, the stabilization of formamide through $\mathrm{H} 2(\mathrm{~N})$... O (water) would not be possible in the $\mathrm{N}$-methyl case. Hence, we can explain the high activity of formamide and its reduction in $\mathrm{N}$-methyl formamide due to the interaction with the water coordinated to $\mathrm{Ca}^{2+}$.

\section{Implications on the catalytic mechanism}

The catalytic mechanism of PLA2 is not adequately developed. In analogy to serine proteases it has been suggested (Verheij et al 1980; Scott et al 1990) that the oxyanion formed from water molecule takes the role of serine hydroxyl group in the catalytic triad. According to this "pseudo-triad" mechanism a third water molecule is involved 
in the catalysis whereas two water molecules coordinated to $\mathrm{Ca}^{2+}$ are substituted by oxygen ligands from the substrate. In this mechanism, ND1 of His48 abstracts a proton from the catalytic water to create an $\mathrm{OH}^{-}$anion, which in turn attacks the ester carbonyl to form a tetrahedral intermediate. There are several difficulties with this model not the least of which is the fact that the catalytic water ("smoking bullet") is not seen in the crystal structure (Thunnisen et al 1990). Further, the $\mathrm{Ca}^{2+}$ and ND1 of His 48 are at a distance of $6.48 \AA$, hence the catalytic water can either be close to $\mathrm{Ca}^{2+}$ or to His48 and not simultaneously to both of them.

The results of the modelling study with the amide provide a novel basis for an alternative catalytic mechanism in which the catalytic water is activated due to its

(a)

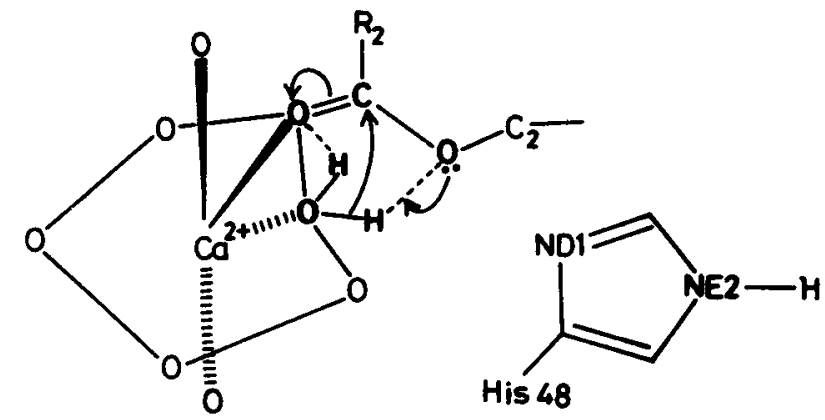

(b)

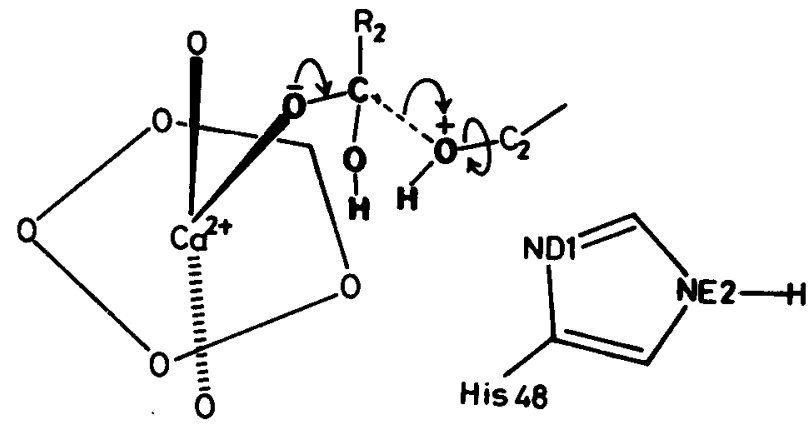

(c)

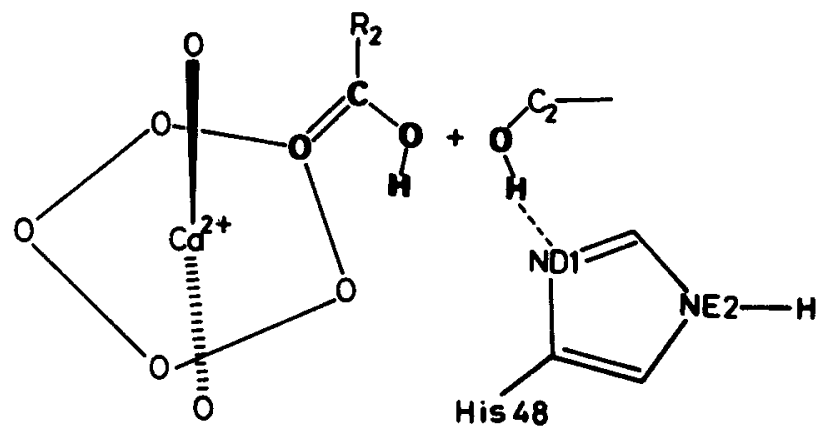

Figure 8. Three major steps postulated in "calcium activated oxyanion" mechanism for the PLA2 catalyzed esterolysis. (a) Attack of the catalytic water on carbonyl carbon; (b) weakening of $\mathrm{C}-\mathrm{O}$ ester linkage; (c) reorientation of ester oxygen-proton to form hydrogen bond with His48 ND1 and cleavage of the ester bond. 
localization in the coordination sphere of calcium. A rationale for this mechanism may be developed from figures $7 a$ and $b$, where the amide is trapped due to $\mathrm{NH} 2 \ldots \mathrm{OH}_{2}$ hydrogen bond interaction. The amide carbonyl oxygen has only displaced the coordinated water and both the amide carbonyl oxygen as well as the water are now coordinated with $\mathrm{Ca}^{2+}$ making it a distorted octa-coordinated calcium. As described below, the relationship between a water molecule polarized by calcium coordination and the localization of the ester ligand form a basis for the "calcium activated oxyanion" mechanism for the PLA2 catalyzed hydrolysis.

A mechanism for the hydrolysis of the $s n$-2-ester bond with a catalytic water activated by $\mathrm{Ca}^{2+}$ is shown in figure 8 . Owing to its coordination to $\mathrm{Ca}^{2+}$, this water is highly polarized and it is suitably positioned to attack the carbonyl group. In the catalytic sequence, the catalytic water attacks the carbonyl carbon making it a near tetrahedral intermediate (figure 8a, top). The proton of the water which was hydrogen bonded to the ester oxygen is transferred to it. These processes weaken the $\mathrm{C}-\mathrm{O}$ ester bond (figure $8 \mathrm{~b}$, middle). The ester bond is completely broken when the proton attached to the ester oxygen orients towards ND1 of His 48 (by $\mathrm{C}_{2}-\mathrm{O}$ bond rotation and the change in the hybridization state of carbonyl $\mathrm{C}$ and ester oxygen) to form a hydrogen bond (figure $8 \mathrm{c}$, bottom). The coordinated water does not move much farther away when the substrate arrives to coordinate with calcium and hence the substrate is acted upon by the water which was originally liganded to $\mathrm{Ca}^{2+}$. This completes the catalytic process. The calcium coordinated oxyanion catalytic mechanism as outlined above is based on the postulate that the coordinated calcium plays a role in the catalytic cycle. This novel suggestion provides a basis to account for several observations that could not be explained by the pseudo-triad mechanism. These include: (a) The role of calcium in the chemical step of the catalytic cycle is not satisfied by substitution by ions like cadmium which have the same size and charge, but prefer different coordination geometries (Yu et al 1993). (b) The catalytic efficiency of the D49N mutant of PLA2 is $\simeq 8 \%$ (Jain et al, to be published) compared to $<0.001 \%$ activity for the corresponding mutant of a serine protease. (c) Hydrated fluoroketone analogs of phospholipids are weak inhibitors. Also the transition state mimics like the $s n$-2-phosphates or phosphonates (cf. figure 3) are about as potent as the amides shown in this figure.

\section{Conclusion}

The $a b$ initio modelling of an amide ligand in the active site of $14 \mathrm{kD}$ PLA2 lead to a unique localization of the amide. The results suggest that during the ligand substitution the hepta coordination shell of calcium in the native enzyme may be distorted to assume an octacoordinated geometry where the carbonyl oxygen as well as a water molecule are within the coordination distance. The catalytically relevant features of this ternary (ECaS) complex include the polarization of the ester carbonyl and of the coordinated water molecule. The geometrical relationship of the water to the ester bond is such that it could act as an electrophile. Thus a catalytic mechanism is developed in which the Ca-polarized water attacks the ester carbonyl. Decomposition of the resulting (near-tetrahedral) transition state complex is facilitated by His48-Asp99 diad.

It is gratifying to obtain such an unique orientation of the ligand from $a b$ initio modelling studies, which can explain important experimental observations. 


\section{Acknowledgements}

We thank Dr P Chakrabarti for sharing his unpublished results on hydrogen bonding ability of an oxygen ligand coordinated to calcium. One of us (KS) gratefully acknowledges the support from the DBT project "Research on Carbohydrates, Cell Surface and Cellular Transport". Hospitality of the Jawaharlal Nehru Centre for Advanced Scientific Research at Bangalore is acknowledged by M K Jain. Financial support for the work was provided by the Council of Scientific and Industrial Research, India (No. 9/326/91-EMR-II), and National Institutes of Health (GM 29703).

\section{References}

Balaji P V 1991 Specificity and mechanism of action of ribonuclease T1-A computer modelling study, Ph D thesis, Indian Institute of Science, Bangalore

Berg O G, Yu B -Z, Rogers J and Jain M K 1991 Biochemistry 307283

Goldstein H 1980 Classical mechanics (Reading, MA: Addison-Wesley) p. 143

Jain M K 1988 Introduction to biomembranes 2nd edn (New York: John Wiley) p. 423

Jain M K and Berg O 1989 Biochim. Biophys. Acta 1002127

Jain M K, de Haas G H, Marecek J F and Ramirez F 1986 Biochim. Biophys. Acta 860475

Jain M K, Gelb M H, Rogers J and Berg O G 1994 Methods in enzymology (in press)

Jain M K, Ghomashchi F, Yu B -Z, Bayburt T, Murphy D, Houck D, Brownell J, Reid J C, Solwiej J E, Wong S M, Mocek U, Jarrell R, Sasser M and Gelb M H 1992 J. Med. Chem. 353584

Jain M K, Rogers J and de Haas G H 1988 Biochim. Biophys. Acta 94051

Jain M K, Rogers J, Hendrickson H S and Berg O G 1993a Biochemistry 328360

Jain M K, Tao W, Rogers J, Arenson C, Eibl H and Yu B -Z 1991 Biochemistry 3010256

Jain M K, Yu B -Z and Berg O G 1993b Biochemistry 3211319

Jain M K, Yuan W and Gelb M H 1989 Biochemistry 284135

Ramachandran G N and Sasisekharan V 1968 Adv. Protein Chem. 23283

Scott D L, White S P, Otwinowski Z, Yuan W, Gelb M H and Sigler P B 1990 Science 2501541

Sekharudu Y C 1985 The modes of binding of carbohydrates to concanavalin A: A computer modelling approach, $\mathrm{Ph} \mathrm{D}$ thesis, Indian Institute of Science, Bangalore

Thunnissen M M G M, Eiso A B, Kalk K H, Drenth J, Dijkstra B W, Kuipers O P, Dikjkman R, de Haas G H and Verheij H M 1990 Nature (London) 347689

Verheij H M, Volwerk J J, Jansen E H J M, Puyk W C, Dijkstra B W, Drenth J and de Haas G H 1980 Biochemistry 19743

Yu B -Z, Berg O G and Jain M K 1993 Biochemistry 326485 